\title{
Real Time Fusion of Range and Light Field Images
}

\author{
Severin S. Todt* \\ University of Siegen, Germany
}

\author{
Christof Rezk Salama \\ University of Siegen, Germany
}

\author{
Andreas Kolb \\ University of Siegen, Germany
}

\begin{abstract}
This poster presents preliminary results of our approach to interactively acquire, process and render corresponding depth and light field information. Our system is based on a handheld image based range sensor in combination with a commodity digital RGB camera facilitating the synchronous acquisition of registered image data. We demonstrate our intermediate system including the ability to simultaneously acquire and process range and greyscale image data and interactively transforming and rendering the data to geometry.
\end{abstract}

\section{Introduction}

With the evolution of 3D shape digitization devices the process of capturing complex geometry and material attributes for single objects or whole scenes is getting simpler, faster and more interactive. Unfortunately existing systems usually suffer from their limited capability to faithfully reproduce real world illumination.

The Lumigraph system [Gortler et al. 1996] introduced the combination of rough geometry representation and high quality light field information to overcome the shortcomings of traditional 3D scanning devices and their subsequent data processing pipeline. Based on a simplified plenoptical function the Lumigraph is capable of rendering arbitrary images of the virtual environment from any vintage point of a virtual camera. The rendering process evolves from light field information images taken in advance. Using silhouette techniques a rough volumetric geometry representation of the observed object is recoverable from the light field images at considerable computational cost. To fully describe the simplified plenoptical function, the camera position is determined for each sample image. For successful camera calibration external markers can be used or great effort can be spent on marker less feature point tracking systems [Koch et al. 2005]. Based on the camera calibration projecting the $2 \mathrm{D}$ light field into $3 \mathrm{D}$ space is a trivial task.

Our approach introduces a real time system merging high resolution light field information and low resolution range data in real time at a consumer price level. The system uses a prototype of a handheld image based PMD (Photonic Mixer Device) range sensor [Kraft et al. 2004] to generate a low resolution $(160 \times 120)$ depth information data set in combination with a commodity digital imaging camera working at VGA resolution $(640 \times 480)$ mounted on top.

\section{Exposition}

The integrated PMD range sensor is capable of capturing pixel aligned depth and greyscale images at interactive frame rates (approx. $10 \mathrm{fps}$ ). The connected RGB camera provides full VGA resolution images of the scene in real time. Image data is acquired constantly at maximum frame rate to maximize interactivity.

In order to achieve high interactivity and ease of use, geometric reconstructions have to be rendered on the fly. Binding the range data images as a vertex texture, the vertices of a highly tessellated grid can be displaced to approximate the captured geometry in real time using shader programs for vertex processing. Binding the light field image as fragment texture the resulting vertex object is textured using standard fragment operations. Normals are calculated using central differences to approximate partial derivatives per vertex. This way artificial lighting is easily added to generate more sophisticated rendering results.

Implementation: In our current system fusion of the range sensor's depth and greyscale image has been implemented as a proof of concept. Data acquisition, data processing and geometric modeling is performed in real time. The range and light field images acquired are fed into a filtering stage. The filtered images are taken as data source for interactive geometry rendering as shown in the Figure. Initial experiments have proven that the relative transformation matrix between the mounted RGB camera and the range sensor can easily be determined using only one known marker. The determined matrix is then used as projective texture matrix to transform the light field image into the range image domain. As a calibration process performed once for the camera setup, projecting the light field data back into 3D space can be performed without significant cost at run time.

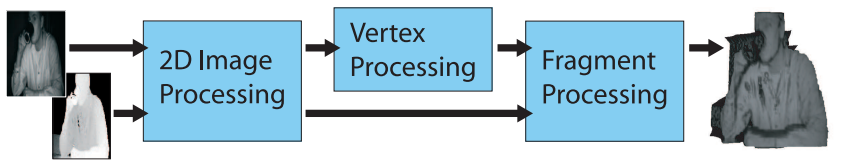

To improve image quality, noisy artifacts in both images can be reduced by linear low pass filters. Convolution is performed using the OpenGL image processing pipeline. More complex image processing tasks must be implemented within the vertex and fragment programs.

Future Directions: In a running system the same single known marker used for camera calibration can be used to determine the camera direction, the last unknown attribute to fully describe the Lumigraph in real time. Capturing and processing a series of image with our system will enhance the idea behind the Lumigraph system for Light Field Rendering in the near future.

\section{References}

Gortler, S. J., Grzeszczuk, R., Szeliski, R., AND COHEN, M. F. 1996. The Lumigraph. In Proceedings of ACM SIGGRAPH 96

Koch, R., Koeser, R., Streckel, B., And Evers-Senne, J.-F. 2005. Markerless Image-based 3D Tracking for Real-Time Augmented Reality Applications. In Workshop on Image Analysis for Multimedia Interactive Services.

Kraft, H., Frey, J., Moeller, T., Albrecht, M., Grothof, M., Schink, B., Hess, H., And Buxbaum, B. 2004. 3D-Camera of High 3D-Frame Rate, Depth-Resolution and Background Light Elimination Based on Improved PMDTechnologies. In OPTO.

*e-mail: \{todt,rezk,kolb\}@fb12.uni-siegen.de 


\section{Real Time Fusion of Range and Light Field Images}

Severin S. Todt Christof Rezk Salama Andreas Kolb

\section{Setup and Operation}

Commodity digital RGB camera mounted on top of PMD range sensor

- Light field information captured at $25 \mathrm{fps}$ by RGB camera

Range data captured at $10 \mathrm{fps}$ with a precision of $\sim 6 \mathrm{~mm}$ at resolution $160 \times 120$ by PMD camera

- Alternative setup captures at $15 \mathrm{fps}$ at resolution 16x64

- Fixed RGB camera installation and easy camera registration enables handheld data aquisition

\section{Camera Registration}

Registration using only one known marker

- AR Toolkit marker detected in light field and PMD grayscale data supplies good guess for camera transformation

- Registration error is depending on the quality of the AR Toolkit Camera

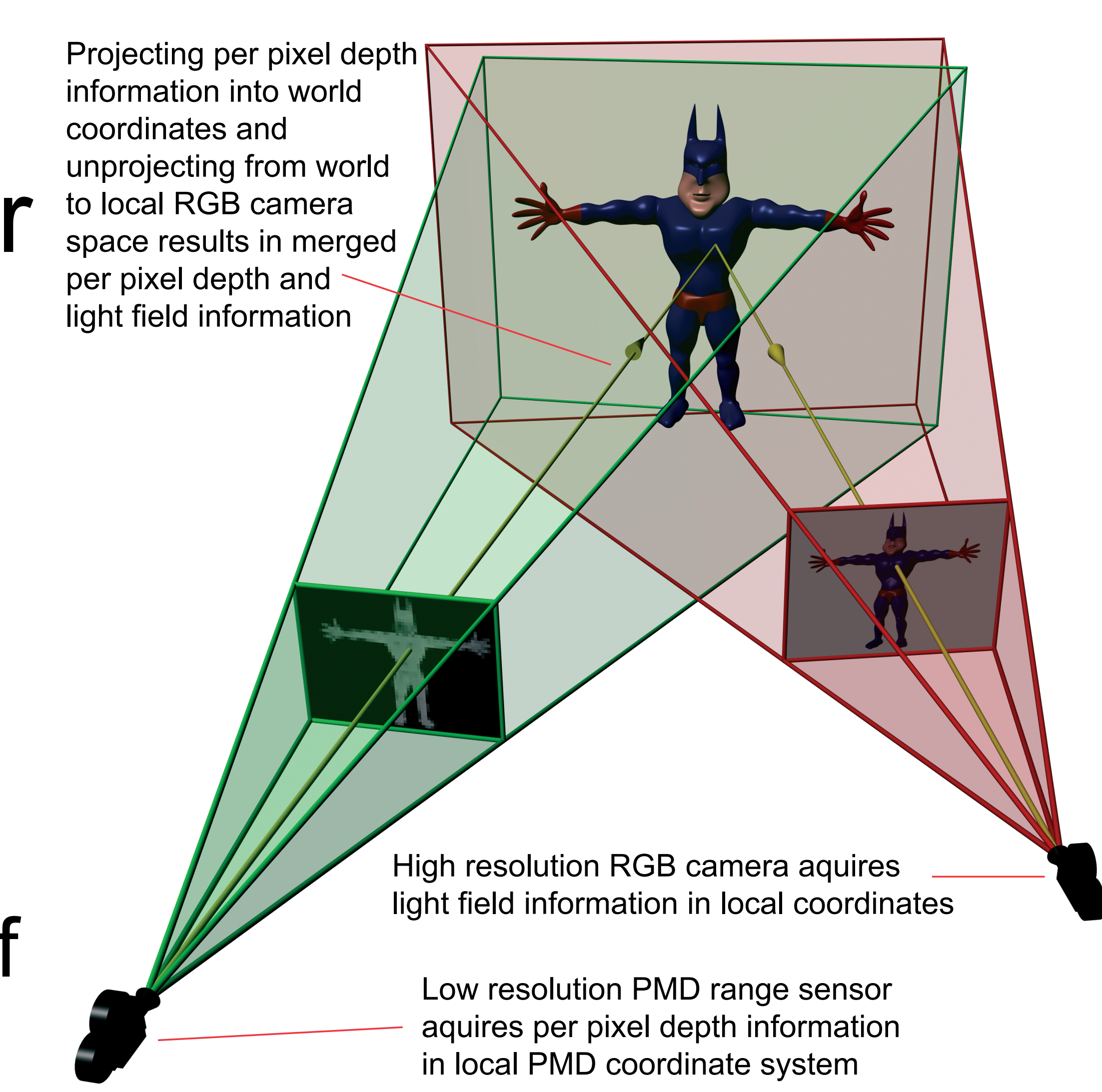

Calibration (error evaluation has not been performed yet)

- Using manually corrected depth information of the marker image, the transformation can be easily adjusted for best fit

- Best camera registration results are measured using a PMD range sensor operating at $160 \times 120$ in combination with a RGB camera setup operating in VGA $(640 \times 480)$ mode

\section{Range and Light Field Fusion}

- Data Fusion based on intrinsic and extrinsic camera parameters

- Camera focal length, pixel dimension, resolution and transformation between cameras required

- Projection from sensor space to world space and unprojection to camera space - Corresponding points are calculated based on the camera registration using intercept theorems
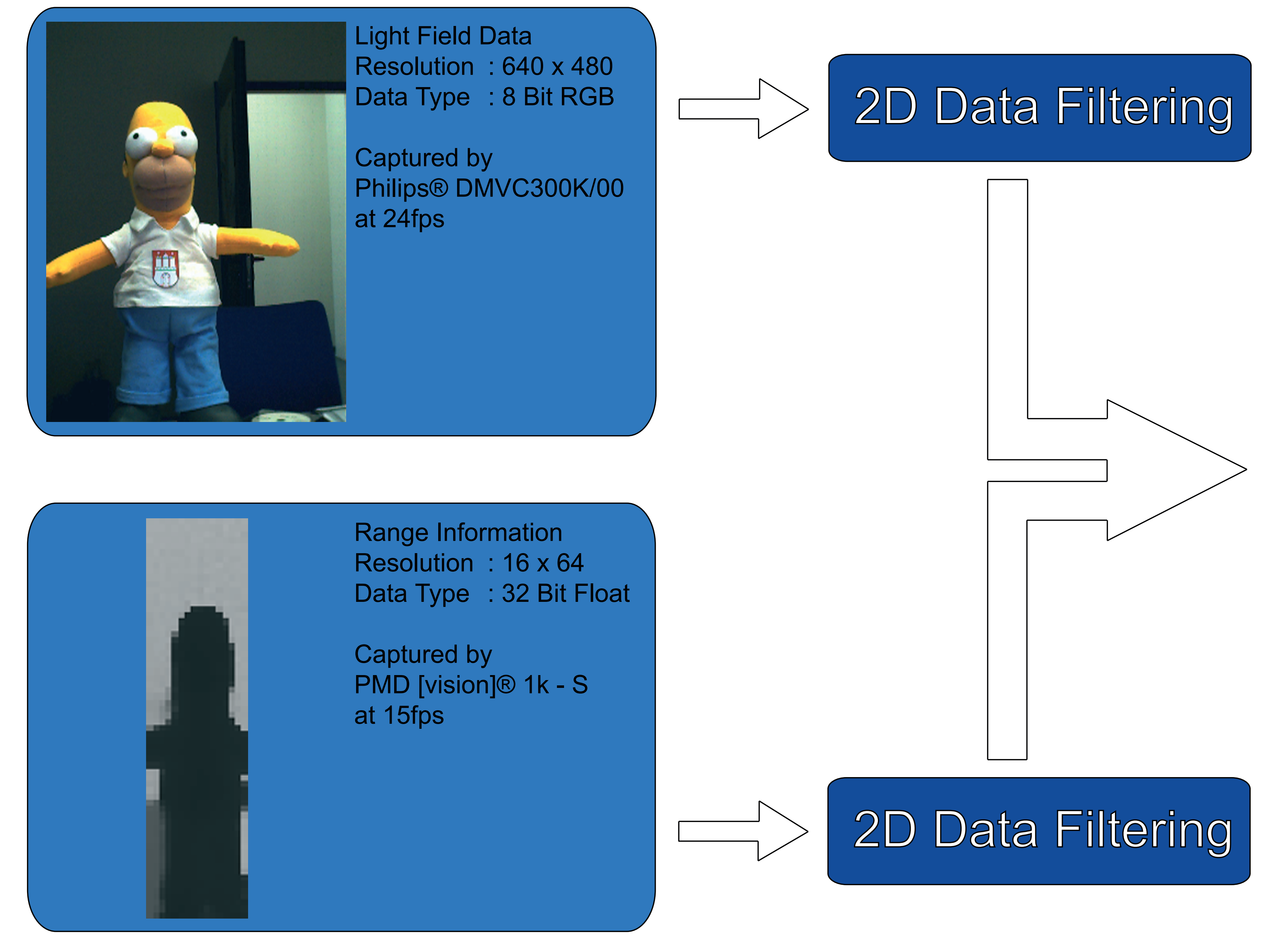

\section{Data Processing}

a GPU based 2D Image Filtering

- Filters and kernels can be applied to source images directly on the GPU or while uploading to the GPU

- Merged range and light field data file format

- Geometry and light field data stored in one image file

- Per pixel depth value stored in alpha channel

- Additional 82 byte header required:

Transform. matrix $16 \times 32$ bit $+2 \times$ (focal length 32 bit, pixel dimensions $2 \times 4$ bit, resolution $2 \times 16$ bit)

\section{Rendering}

Vertex texture for per vertex displacement

- Range data images as vertex texture

- Per pixel displacement mapping for geometry reconstruction in real time

- Light field image as standard fragment texture

- Per vertex normals for artificial lighting

- Normals calculated on the fly using central differences
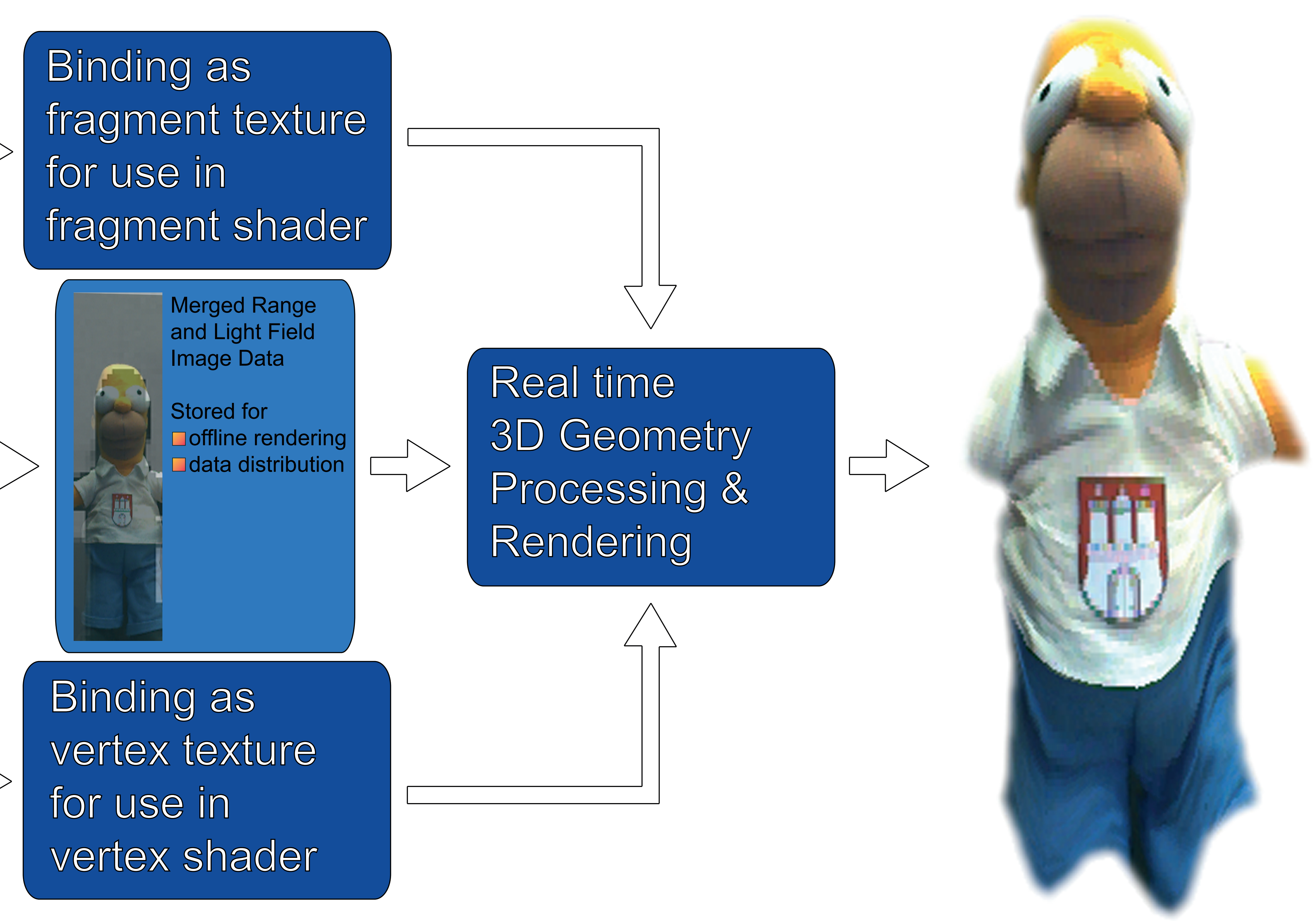

\section{Results and Future Directions}

Running System capable of simultanously aquiring registered depth and light field data

- Identifiying one known marker in the scene the Lumigraph is fully describable in real time

Emerging precision and resolution for new directions

- Higher resolution for more accurate geometry reconstruction and rendering 